\title{
Maraviroc Intensification in HIV-1 Infected Patients with Persistant Low-level Viremia
}

Damien Le Dû́, ${ }^{1,2 *}$, Dhiba Marigot-Outtandy ${ }^{1,2}$, Dominique Mathez ${ }^{1}$, Caroline Dupont ${ }^{1,3}$, Mamadou Saliou Sow ${ }^{1}$, Françoise Borsa-Lebas ${ }^{4}$, Elisabeth Rouveix ${ }^{1,3}$ and Pierre de Truchis ${ }^{1,3}$

${ }^{1}$ Groupe Hospitalo-universitaire Paris lle de France Ouest AP-HP, France

${ }^{2} \mathrm{CH}$ Bligny, Briis-sous-Forges, France

${ }^{3}$ COREVIH lle de France Ouest, France

${ }^{4} \mathrm{CHU}$ Rouen, France

\begin{abstract}
HIV-1 persistent low-level-viremia under 500 copies RNA/ml (PLV) is associated with an increased risk of virological progression and resistance. We performed a study on 18 HIV-1 treated patients who received ARV treatment intensification with maraviroc (MVC) because of PLV during the 6 last months or more. During the 12 months following intensification, CD4 T-cells increased by an average of $104 / \mathrm{mm} 3$. Percentage of patients with a plasmatic viral load under 50 copies $/ \mathrm{ml}$ increased regularly since intensification to reach $71 \%$ after 12 months of follow-up. MVC intensification could improve virological and immunological responses in case of PLV.
\end{abstract}

Keywords: HIV-1; Maraviroc, ARV intensification; Low-level viremia

\section{Introduction and Objective}

The goal of antiretroviral therapy is to achieve viral suppression, which is determined by HIV-1 plasma viral load (VL) below 50 copies/ $\mathrm{ml}$. It has been reported that a significant proportion of patients under highly active antiretroviral therapy (HAART) according to the results of genotyping tests, present persistent low-level viremia (PLV) defined by sustained VL between 50 and 500 HIV-RNA copies $/ \mathrm{ml}$ [1]. Resistance testing has been shown to be an effective predictor of future virological failure [2]. However, most commercial resistance tests can only be performed when VL is above a minimum of 200-500 copies/ml. Ultrasensitive HIV-1 genotyping for patients with PLV has shown that significant resistance mutations were often present [3]. Consequences of PLV remain unclear but several studies shows that incomplete viral suppression leads to the accumulation of resistance mutations with a concomitant increased risk of virological progression and clinical deterioration, and compromised future treatment options [4-11]. Moreover, antiretroviral-treated individuals with PVL exhibit significant increase in overall immune activation [12]. The threshold of PLV as predictive factor of disease progression varies upon studies. Treatment optimization in HIV-1 infected patients with PLV, based on patient therapeutic history and genotypic resistance profiles, significantly improve viral suppression [13]. The best intervention to achieve full virological suppression in this population remains unclear. Intensification with maraviroc (MVC) was previously studied in patients with VL under threshold, without efficacy to reduce immunological activation, but was not evaluated in HIV-1 infected patients with PLV. To determine whether this optimization can improve viral suppression, we retrospectively analyzed HIV-1infected patients, who received MVC-intensification because of PLV in spite of on-going HAART. We aim to describe the virological and immunological impact of adding MVC in this population.

\section{Material and Methods}

We performed a multicentric retrospective study between January 2012 and October 2013, in three French academic Hospitals. We included HIV-1 treated patients whose treatment had been intensified by MVC because of PLV defined by detectable VL below 500 HIV-RNA copies/ml for more than 6 months. A minimum of three VL measures on this period was required for inclusion in the study. Treatment adherence, evaluated by patient's interview and drug therapeutic monitoring, was considered as satisfying by the patient's physician. On-going antiretroviral treatments before intensification were effective regarding to the results of genotyping tests. MVC was added to the on-going antiretroviral regimen, without any other therapeutic modification.

For each patient, we collected demographical data, comorbidities, therapeutic and immuno-virological history including the results of genotyping tests and viral tropism. We also collected results of blood samples for VL and CD4 T-cells (count and percentage), at least every two months for the next six months after intensification, and at 12 months.

Plasma VL was evaluated by Roche Cobas Taq man HIV-1 v.2.0 test with a detection limit of 20 copies $/ \mathrm{ml}$. Coreceptor usage was determined from RNA genotypic analysis of the V3 env. region sequence by Geno2Pheno2 algorithm. Quantitative data are presented as median [IQR] values, unless otherwise stated in text. Wilcoxon signed-rank test was used to test the paired differences in values obtained from baseline to each step of study for significance. A Chi-2 test adapted for small numbers was used to test the difference in rates of VL under threshold between the different groups.

\section{Results}

Eighteen patients were included in analysis. Fifteen (83\%) were men, with mean age 46 years old (range 3 - 65). Two were hepatitis $C$ and none hepatitis B co-infected. Nine (50\%) patients were stage C (AIDS)

*Corresponding author: Damien Le Dû, CH Bligny, 91640, Briis-sous-Forges, France, Tel: 0033169263191; Fax: 0033169263095; E-mail: d.ledu@chbligny.fr

Received April 24, 2016; Accepted May 12, 2016; Published May 18, 2016

Citation: Le Dû D, Marigot-Outtandy D, Mathez D, Dupont C, Sow MS, et al. (2016) Maraviroc Intensification in HIV-1 Infected Patients with Persistant Lowlevel Viremia. J AIDS Clin Res 7: 578. doi:10.4172/2155-6113.1000578

Copyright: @ 2016 Le Dû et al. This is an open-access article distributed under the terms of the Creative Commons Attribution License, which permits unrestricted use, distribution, and reproduction in any medium, provided the original author and source are credited. 
according to the WHO classification. Median CD4 T-cells nadir was 83 (37-137) $/ \mathrm{mm}^{3}$. Median time since HIV diagnosis was 13 (5-20) years and median duration of PLV before MVC-intensification was 9 (6-16) months. Background antiretroviral regimens before intensification included 2 or more nucleoside reverse transcriptase inhibitors for 16 (89\%) patients, boosted protease inhibitor for $11(61 \%)$, raltegravir for $11(61 \%)$, and/or non nucleoside reverse transcriptase inhibitor for $8(44 \%)$. Fourteen $(78 \%)$ patients had confirmed CCR5-receptor tropism, one had dual/mixed (CCR5- and CXCR4-receptor) tropic viruses and 3 undetermined tropisms.

At time of MVC intensification, all patients had detectable plasmatic VL with a median of 70 (47-139) copies/ml. On second month (M2), M4, M6 and M12, respectively $31 \%, 38 \%, 44 \%$, and $57 \%$ of patients reached undetectable VL below $20 \mathrm{HIV}$-RNA copies/ $\mathrm{ml}$ with a median VL of respectively 104 (39-145), 39 (19-109), 39 (19-79), and 23 (1978) copies $/ \mathrm{ml}$. Since intensification, percentage of patients with VL under 50 copies $/ \mathrm{ml}$ increased regularly to reach $71 \%(\mathrm{P} \leq 0.05)$ after M12 of follow-up (Figure 1). Moreover, according to the Wilcoxon signed-rank test, VL decreased significantly from baseline (M0) to M12 $(\mathrm{P} \leq 0.05)$. At baseline, average CD4 T-cells count was $520 / \mathrm{mm} 3$ at time of MVC intensification and reached $624 / \mathrm{mm} 3$ six months later.

\section{Discussion}

In our study, MVC intensification in patients with PLV has a positive impact on VL evolution: twelve months after intensification, $71 \%$ of the analyzed patients reached a VL below 50 copies $/ \mathrm{ml}$ versus $18 \%$ at the time of therapeutic intervention. As a CCR5 antagonist, MVC specifically inhibits the replication of R5-tropism viruses by blocking viral entry. Before intensification, none of our patients had antiretroviral treatment targeting viral entry, so addition of this new therapeutic class to the current antiretroviral treatment is likely to explain the benefit in virological efficacy in these patients. In previous studies, efficacy of antiretroviral intensification with other strategies in patients with PLV were not as convincing: antiretroviral intensification using inhibitors of HIV-1 reverse transcriptase (efavirenz) or protease (atazanavir-ritonavir or lopinavir-ritonavir) did not lower HIV-1 RNA levels in a first study [14]. Raltegravir (RAL) did not prove any effect for reducing the plasmatic VL in another study but RAL-adding period was only 28 days and only 10 patients were included [15]. These results indicated that additional inhibition of either the reverse-transcription, the protease-cleavage steps in viral replication, or the DNA-integration steps does not further inhibit HIV-1 production in patients with persistent viremia in most of cases.

Our study was retrospective and included a limited number of patients. To our knowledge, no previous study observed the virological effect of MVC intensification in cases of detectable viral load with PLV $>50$ copies $/ \mathrm{ml}$. However, in a pilot open-label phase 2 clinical trial, patients with stable HAART regimen receiving MVC intensification for 48 weeks, showed a non-significant reduction of latent reservoir $(\mathrm{p}=0.068)$ and no effect on plasma residual viremia was observed. Moreover, no changes were detected in CD4 or CD8 T-cells counts [16].

Several other trials have studied the effects of treatment intensification by maraviroc or raltegravir on residual viral replication and immunological activation in patients with undetectable VL $(<50$ copies/ml) under antiviral therapy; a significant effect on residual viral replication was never assessed, while results on inflammatory activation are variable, partial, and inconsistent across studies [17].

A recent study of switch therapy with raltegravir and maraviroc for patients with $\mathrm{VL}<50$ copies/ml has not demonstrated virological or immunological improvement [18]; but, our patients had a higher level of viral replication before intensification with maraviroc, and this should explain the better results observed.

In our study, CD4 T-cells count increased during the first 6 months after MVC intensification, from an average of $520 / \mathrm{mm} 3$ to $624 / \mathrm{mm} 3$. Some studies have already explored the immunological effect of MVC intensification for patients with low immunological response to HAART. A pilot trial (ACTG A5256) of adding maraviroc

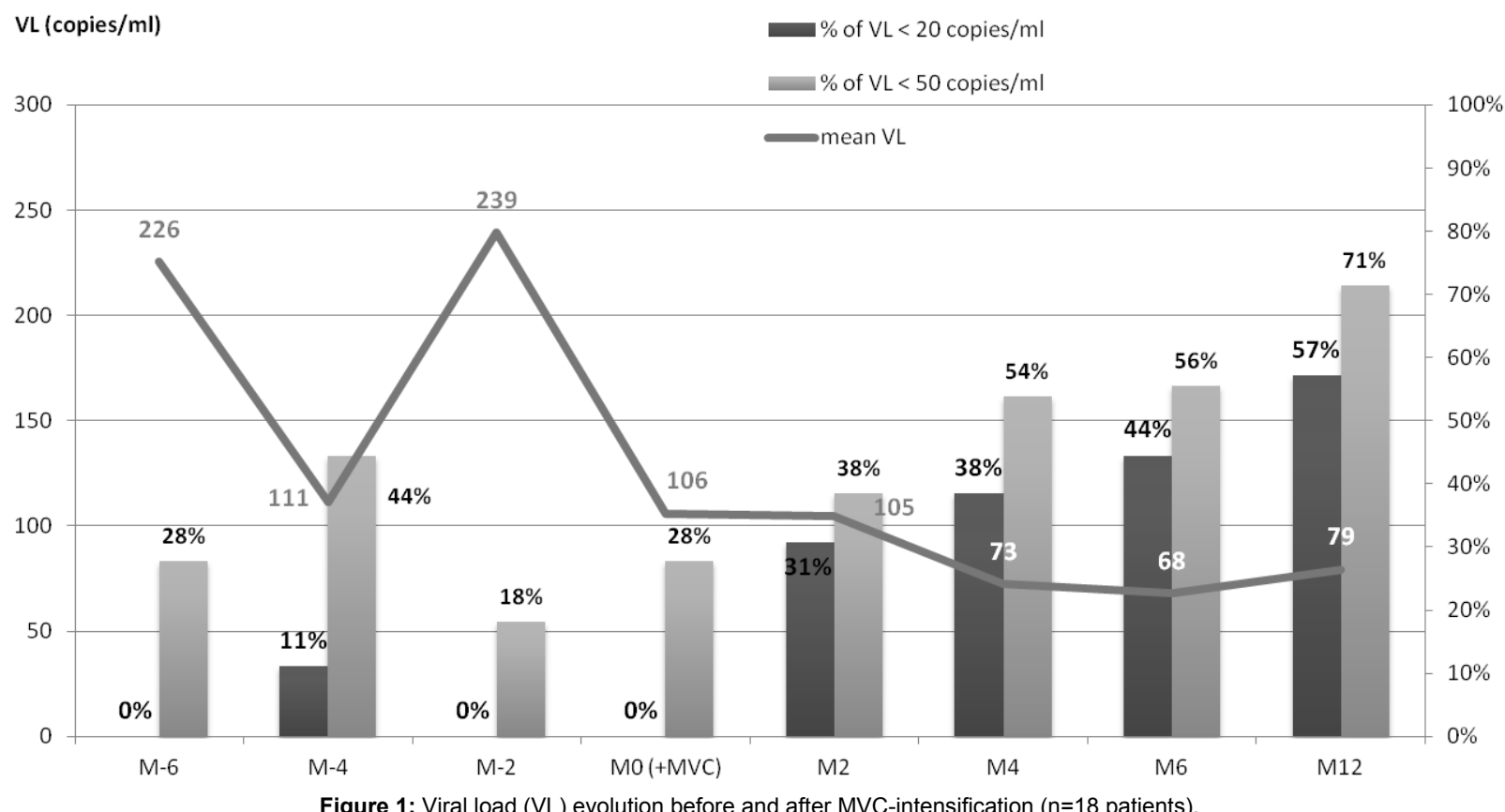


for 24 weeks to suppressive antiretroviral therapy in 34 patients presenting suboptimal CD4 T-cell recovery despite sustained virologic suppression, did not demonstrate an increase in CD4 T-cell counts of at least 20 cells/ $\mu \mathrm{L}$ [19]. But, in MARIMUNO study, 60 patients with $\mathrm{CD} 4<350 / \mathrm{mm}^{3}$ and a CD4-slope $<50$ cells $/ \mathrm{mm}^{3} /$ year in spite of sustained virological response $(<50 \mathrm{copies} / \mathrm{ml})$ over the last two years while receiving a stable HAART, were included for 24 weeks of MVC intensification. The median CD4 slope difference from baseline was +22.6 cells $/ \mathrm{mm}^{3} /$ year $(\mathrm{p}=0.08)$. Slope evolution was not different according to baseline tropism, CD4 nadir, or ongoing HAART regimen [20]. Whereas patients included in our study experienced a CD4 T cells count increase, the results of previous studies analyzing immunological impact of MVC adding to a previous regimen are discordant.

\section{Conclusion}

However limited and retrospective, our study shows a beneficial virological effect of treatment intensification with maraviroc in most of the patients with persistent low level viral replication under antiretroviral therapy. These findings need to be confirmed by further prospective and randomized trials.

\section{References}

1. Pozniak A, Gupta RK, Pillay D, Arribas J, Hill A (2009) Causes and consequences of incomplete HIV RNA suppression in clinical trials. HIV Clin Trials 10: 289-298.

2. Powderly WG, Saag MS, Chapman S, Yu G, Quart B, et al. (1999) Predictors of optimal virological response to potent antiretroviral therapy. AIDS 13: 18731880.

3. Nettles RE, Kieffer TL, Simmons RP, Cofrancesco J Jr, Moore RD, et al. (2004) Genotypic resistance in HIV-1-infected patients with persistently detectable low-level viremia while receiving highly active antiretroviral therapy. Clin Infect Dis 39: 1030-1037.

4. Cohen C (2009) Low-level viremia in HIV-1 infection: consequences and implications for switching to a new regimen. HIV Clin Trials 10: 116-124.

5. Laprise C, de Pokomandy A, Baril JG, Dufresne S, Trottier H (2013) Virologic failure following persistent low-level viremia in a cohort of HIV-positive patients: results from 12 years of observation. Clin Infect Dis 57: 1489-1496.

6. Taiwo B, Gallien S, Aga E, Ribaudo H, Haubrich R, et al. (2011) Antiretroviral drug resistance in HIV-1-infected patients experiencing persistent low-level viremia during first-line therapy. J Infect Dis 204: 515-520.

7. Gonzalez-Serna A, Min JE, Woods C, Chan D, Lima VD, et al. (2014) Performance of HIV-1 drug resistance testing at low-level viremia and its ability to predict future virologic outcomes and viral evolution in treatment-naive individuals. Clin Infect Dis 58: 1165-1173.

8. Swenson LC, Min JE, Woods CK, Cai E, Li JZ, et al. (2014) HIV drug resistance detected during low-level viraemia is associated with subsequent virologic failure. AIDS 28: 1125-1134.

9. Aleman S, Söderbärg K, Visco-Comandini U, Sitbon G, Sönnerborg A (2002 Drug resistance at low viraemia in HIV-1-infected patients with antiretroviral combination therapy. AIDS 16: 1039-1044.

10. Sungkanuparph S, Groger RK, Overton ET, Fraser VJ, Powderly WG (2006) Persistent low-level viraemia and virological failure in HIV-1-infected patients treated with highly active antiretroviral therapy. HIV Med 7: 437-441.

11. Delaugerre C, Gallien S, Flandre P, Mathez D, Amarsy R, et al. (2012) Impact of low-level-viremia on HIV-1 drug-resistance evolution among antiretroviral treated-patients. PLoS One 7: e36673.

12. Karlsson AC, Younger SR, Martin JN, Grossman Z, Sinclair E, et al. (2004) Immunologic and virologic evolution during periods of intermittent and persistent low-level viremia. AIDS 18: 981-989.

13. McConnell MJ, Mier-Mota J, Flor-Parra F, Martinez-Fernandez FJ, LopezCortes LF, et al. (2011) Improved viral suppression after treatment optimization in HIV-infected patients with persistent low-level viremia. J Acquir Immune Defic Syndr 58: 446-449.

14. Dinoso J, Kim SY, Wiegand AM, Palmer SE, Gange SJ, et al. (2009) Treatment intensification does not reduce residual HIV-1 viremia in patients on highly active antiretroviral therapy. Proc Natl Acad Sci U S A 106: 9403-9408.

15. McMahon D, Jones J, Wiegand A, Gange SJ, Kearney M, et al. (2010) Shortcourse raltegravir intensification does not reduce persistent low-level viremia in patients with HIV-1 suppression during receipt of combination antiretroviral therapy. Clin Infect Dis 50: 912-919.

16. Gutiérrez C, Díaz L, Vallejo A, Hernández-Novoa B, Abad M, et al. (2011) Intensification of antiretroviral therapy with a CCR5 antagonist in patients with chronic HIV-1 infection: effect on T cells latently infected. PLoS One 6: e27864.

17. Llibre JM, Buzón MJ, Massanella M, Esteve A, Dahl V, et al. (2012) Treatment intensification with raltegravir in subjects with sustained HIV-1 viraemia suppression: a randomized 48-week study. Antivir Ther 17: 355-364.

18. Campillo-Gimenez L, Assoumou L, Valantin MA, Pajanirassa P, Villemonteix J, et al. (2015) Switch to maraviroc/raltegravir dual therapy leads to an unfavorable immune profile with low-level HIV viremia. AIDS 29: 853-856.

19. Wilkin TJ, Lalama CM, McKinnon J, Ghandi RT, Lin N, et al. (2012) Apilot tria of adding maraviroc to suppressive antiretroviral therapy for suboptimal CD4+ T-cell recovery despite sustained virologic suppression, ACTG A5256. J Infect Dis 206: 534-542.

20. Cuzin L, Trabelsi S, Delobel P, Barbuat C, Reynes J, et al. (2012) Maraviroc intensification of stable antiviral therapy in HIV-1-infected patients with poo immune restoration: MARIMUNO-ANRS 145 study. J Acquir Immune Defic Syndr 61: 557-564. 